\title{
Robust Multiple Watermarking Technique for Medical Applications using DWT, DCT and SVD
}

\author{
Imane Assini, Abdelmajid Badri, Khadija Safi, Aicha Sahel, Abdennaceur Baghdad \\ EEA\&TI laboratory Faculty of Sciences and Techniques (FSTM), Hassan II University Casablanca, \\ BP 146, Mohammedia, 20650 Morocco \\ assini.media@gmail.com
}

\begin{abstract}
The security of medical data plays an important role in preserving their integrity and confidentiality. This paper presents a robust multiple watermarking algorithm based on a combination of discrete wavelet transform (DWT), discrete cosine transform (DCT) and singular value decomposition (SVD).

In this proposed method, we have inserted three watermark images (a patient image, a doctor image and a fingerprint of the patient) into a single cover medical image. In the insertion phase, the original image is decomposed into the third level of DWT, and then the DCT and SVD transforms are applied on the selected sub bands ( $\mathrm{HH} 1, \mathrm{HH} 2$, and $\mathrm{HH} 3)$. The first watermark goes through the same steps that the original image. Moreover, the second and the third watermarks are decomposed until the second level and the first level by DWT respectively and subsequently transformed by SVD. The singular values of the three watermarks are inserted into the singular values of the original image. The experimental results show that the proposed method gives better performance in terms of capacity, quality and robustness against various attacks compared to other reported techniques.
\end{abstract}

Keywords-multiple watermarking, medical image, DWT, DCT, DWT.

\section{Introduction}

Telemedicine plays an important and vital role in the aid to the diagnosis and decision making; it is a wellknown application that facilitates the transfer of the enormous amount of medical data[1].

Our approach is situated within this frame and it consists on conceiving a control and monitoring system in order to protect medical data spread between hospital centers. The idea was then to think to the digital watermarking. Digital watermarking is a technique of embedding information into a medical image (Magnetic Resonance (MRI), Computed Tomography (CT), Mammography (MG)...) for various purposes such as the fight against fraud and the protection of copyright. Recently, multiple image watermarking appeared as a solution to enhance the confidentiality and security of the inserted watermarks.

In the literature, there are a number of contributions using the multiple image watermarking such as:

In [2], the method proposed by the authors is based on multiple watermarking for healthcare application which combines DWT, DCT, and SVD. In the embedding process, two watermarks are inserted into the cover medical image after their decomposition up to the third level by DWT and their transformation by 
Imane Assini, Abdelmajid Badri, Khadija Safi, Aicha Sahel, Abdennaceur Baghdad. Robust Multiple Watermarking Technique for Medical Applications using DWT, DCT and SVD. Transactions on Machine Learning and Artificial Intelligence, Vol 5 No 4 August (2017); p: 463-472

DCT and SVD respectively. The experimental results show that this contribution gives an excellent performance in terms of robustness and imperceptibility. In [3], the authors present a secure multiple watermarking technique based on the combination of DWT, DCT, and SVD for medical images. This proposed method uses three watermarks, one medical image and two text watermarks. This watermarking scheme gives a good efficiency for security, capacity, imperceptibility and robustness.

In [4], the authors proposed a hybrid technique for medical images based on Non sub sampled contourlet transform (NSCT) which combines discrete cosine transform (DCT), Multiresolution Singular value decomposition(MSVD) and Arnold transform. Three watermarks (two images and text watermark) are embedded into a single cover medical image. Experimental results approve that this proposed method produces high robustness against various attacks.

The steps of our paper are as follows: Step 1 presents the techniques used in our approach, in the step 2 the hybrid algorithm DWT-DCT-SVD is proposed, in the step 3 the experimental results are presented and in the final step a conclusion of the work is done.

\section{Used techniques}

The proposed multiple watermarking is based on DWT, DCT, and SVD. The choice of these three techniques it's focused on their properties, DWT allows a good location in the time and the frequency domain and it's better to identify appropriate data to human perception (HVS)[3], DCT has a good perceptual invisibility, an acceptable robustness, and a reasonable complexity[2, 5]. And finally, the important properties of the SVD is that even if one applies a small variation in the singular values it does not affect the quality of the image [6] as well as the singular values of an image have a great stability.

\subsection{Discrete Wavelet Transform (DWT)}

The discrete wavelet transform (DWT) [7] is a multiresolution description which consists of decomposing image into four frequency representations. These four representations are called sub-bands LL (approximation), LH (vertical), $\mathrm{HL}$ (horizontal) and $\mathrm{HH}$ (diagonal).

\subsection{Discrete Cosine Transform (DCT)}

The discrete cosine transform (DCT) [8] is a function that transforms the representation of a data from the spatial domain to the frequency domain. The energy of the image is concentrated in the sub band of the low frequencies which contains the most important visual part of the image. Most watermarking techniques based on DCT embed the watermark in the middle frequency sub-bands because attacks can delete the high-frequency components of the image.

\subsection{Singular Value Decomposition (SVD)}

The singular value decomposition (SVD) [6] of an image is represented by a matrix which can be factored into a product of two orthogonal matrices and a diagonal matrix.

The decomposition of an image into SVD can be written as (1):

$$
\mathrm{I}=\mathrm{U} * \mathrm{~S} * \mathrm{VT}
$$

Where $S$ is a matrix whose diagonal terms are positive and orderly decreasing, and all other terms being zero. The singular values represent the energy of the image. 


\section{Performance measures}

The Imperceptibility of the proposed watermarking system is evaluated by the calculating the Peak Signal to Noise Ratio(PSNR) (3) which is based on the mean square error (MSE) defined by (2)[9]:

$$
M S E=\frac{1}{m n} \sum_{i=1}^{m} \sum_{j=1}^{n}\left[X(i, j)-X^{\prime}(i, j)\right]^{2}
$$

Where $X$ and $X^{\prime}$ are the cover medical image and the watermarked medical image respectively of size $\mathrm{m} x$ $\mathrm{n}$.

$$
P S N R=10 \log \left[\frac{255}{M S E}\right]
$$

Furthermore, the robustness of the watermarking scheme is measured by the correlation between the reference watermark and the extracted watermark by (4)[9]:

$$
N C=\frac{\sum_{i=1}^{M} \sum_{j=1}^{N}\left(I(i, j) * I^{\prime}(i, j)\right)}{\sqrt{\sum_{i=1}^{M} \Sigma_{j=1}^{N} I(i, j)^{2} * \sum_{i=1}^{M} \sum_{j=1}^{N} I \prime(i, j)^{2}}}
$$

Where $I(i, j)$ is a pixel of the original watermark of size $M \times N$ and $I^{\prime}(i, j)$ is a pixel of the extracted watermark of size $\mathrm{M} \times \mathrm{N}$.

\section{Proposed method}

The proposed method DWT-DCT-SVD has two steps, embedding and extracting process illustrated in figures 1 and 2.

\subsection{Embedding process}

1. Decomposing the cover medical image into the third level DWT transform using Haar wavelet and then select $\mathrm{HH} 1, \mathrm{HH} 2$, and $\mathrm{HH} 3$.

2. Apply step1 to the first watermark image 'Patient image' and select $\mathrm{HH}_{3 \text { patient. }}$

3. Apply the second and the first level of DWT to the second and the third watermark images 'Doctor image' and 'Fingerprint' respectively and then select $\mathrm{HH}_{2 \text { doctor }}$ and $\mathrm{HH}_{1 \text { fingerprint. }}$

4. Apply DCT on the selected sub bands $\mathrm{HH} 1, \mathrm{HH} 2, \mathrm{HH} 3$ and $\mathrm{HH} 3$ patient of the original medical image and watermark image1 'Patient image' respectively and then apply SVD on DCT of $(\mathrm{HH} 1, \mathrm{HH} 2$, $\mathrm{HH} 3$ and $\mathrm{HH} 3$ patient) and on $\mathrm{HH} 2$ doctor and HH1fingerprint of the second and the third watermark images to get :

$$
\begin{gathered}
\boldsymbol{A}_{\boldsymbol{i}}=\boldsymbol{U}_{\boldsymbol{i}} \boldsymbol{S}_{\boldsymbol{i}} \boldsymbol{V}_{\boldsymbol{i}}^{\boldsymbol{T}} \mathrm{i}=\mathrm{HH}_{1}, \mathrm{HH}_{2} \text { and } \mathrm{HH}_{3} \\
\boldsymbol{w}_{\boldsymbol{i}}=\boldsymbol{U}_{\boldsymbol{j}} \boldsymbol{S}_{\boldsymbol{j}} \boldsymbol{V}_{\boldsymbol{j}}^{\boldsymbol{T}} \mathrm{j}=\text { Patient image, Doctor image and Fingerprint }
\end{gathered}
$$

5. Modify the singular values of $\mathrm{HH} 3$ of the cover medical image with the singular values of $\mathrm{HH} 3$ patient of watermark image 1 'Patient image', the singular values of $\mathrm{HH} 2$ of the cover medical image with the sigular values of $\mathrm{HH}_{2 \text { doctor }}$ of watermark image 2 'Doctor image' and finaly the singular values of $\mathrm{HH}_{1}$ of the cover medical image with the singular values of $\mathrm{HH}_{1 \text { fingerprint }}$ by (7):

$\alpha$ :Scaling factor

$$
A_{w a t i}=S_{i}+\alpha * S_{j}
$$

6. Apply inverse DCT and then inverse DWT to get the watermarked medical image. 


\subsection{Extracting process}

1. Decomposing the cover medical image into the third level DWT transform using Haar wavelet and then select $\mathrm{HH}_{1}, \mathrm{HH}_{2}$, and $\mathrm{HH}_{3}$.

2. Apply step1 to the first watermark image 'Patient image' and select $\mathrm{HH}_{3 \text { patient. }}$

3. Apply the second and the first level of DWT to the second and the third watermark images 'Doctor image' and 'Fingerprint' respectively and then select $\mathrm{HH}_{2 \text { doctor }}$ and $\mathrm{HH}_{1 \text { fingerprint }}$
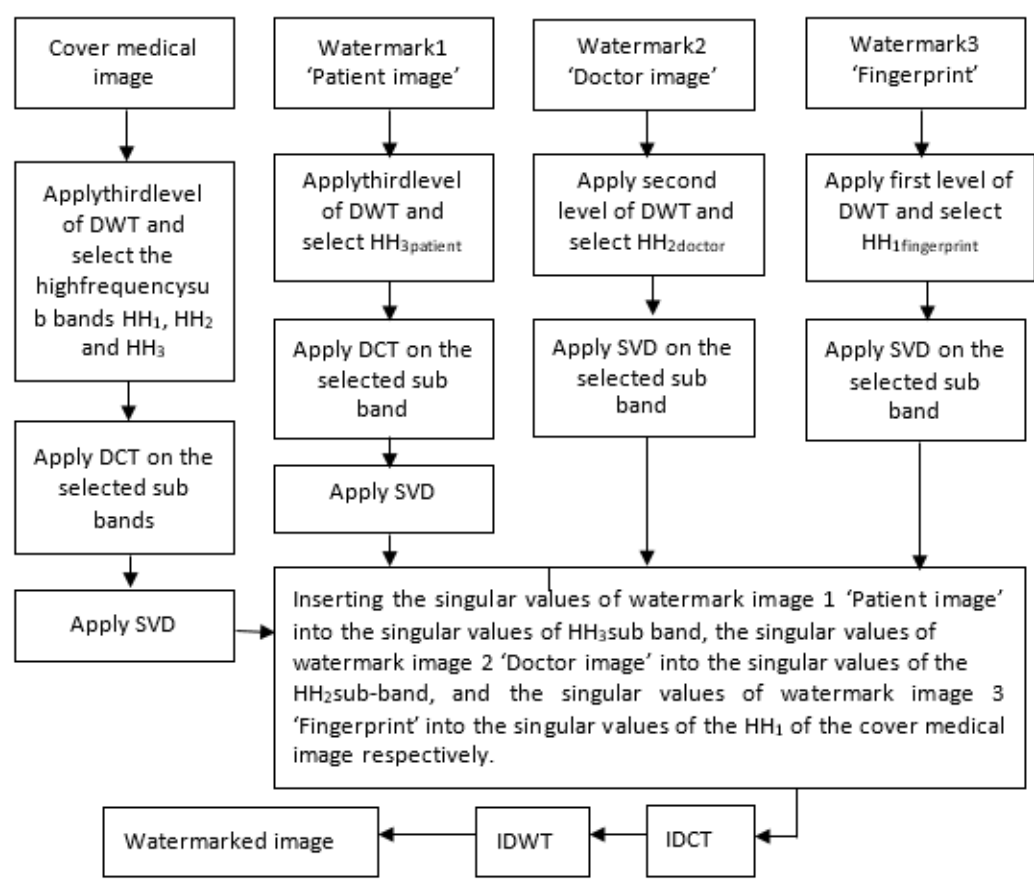

Figure 1. Embedding process using DWT-DCT-SVD
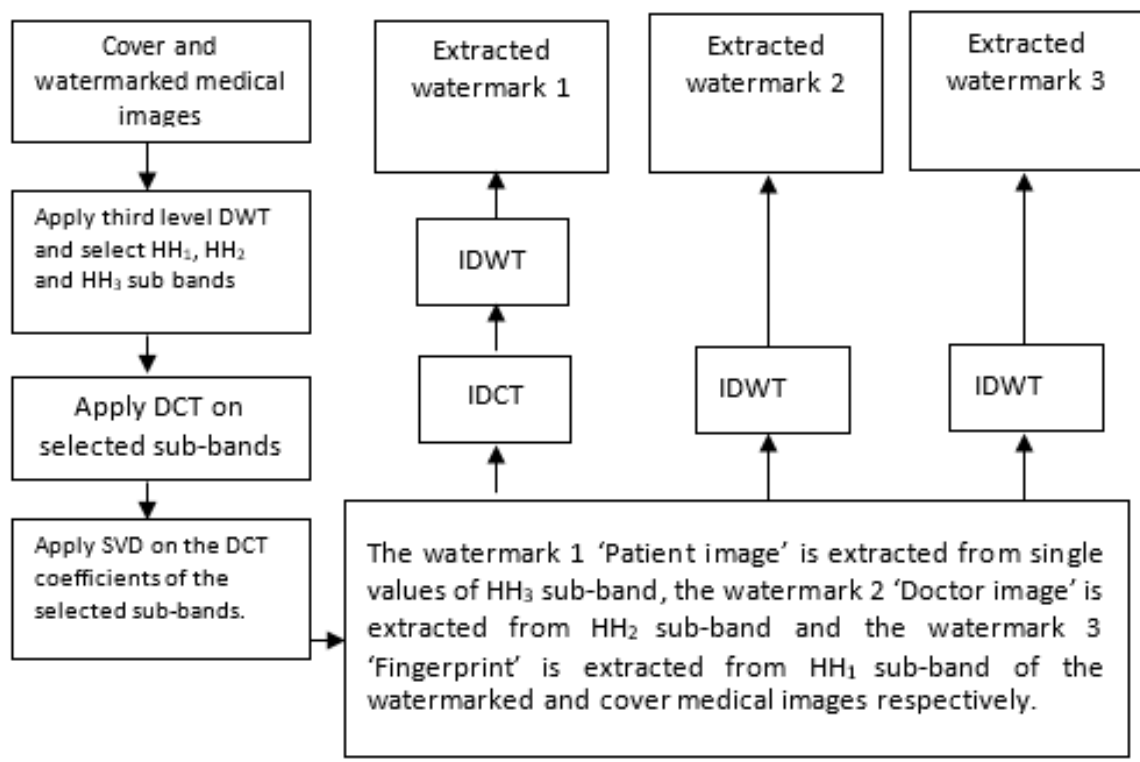

Figure 2. Extracting process using DWT-DCT-SVD 
4. Apply DCT on the selected sub bands $\mathrm{HH}_{1}, \mathrm{HH}_{2}, \mathrm{HH}_{3}$ and $\mathrm{HH}_{3 p a t i e n t}$ of the original medical image and watermark image1 'Patient image' respectively and then apply SVD on DCT of $\left(\mathrm{HH}_{1}, \mathrm{HH}_{2}\right.$, $\mathrm{HH}_{3}$ and $\mathrm{HH}_{3 \text { patient }}$ ) and on $\mathrm{HH}_{2 \text { doctor }}$ and $\mathrm{HH}_{1 \text { fingerprint }}$ of the second and the third watermark images to get :

$$
\begin{gathered}
\qquad \boldsymbol{A}_{\boldsymbol{i}}=\boldsymbol{U}_{\boldsymbol{i}} \boldsymbol{S}_{\boldsymbol{i}} \boldsymbol{V}_{\boldsymbol{i}}^{\boldsymbol{T}}, \mathrm{i}=\mathrm{HH}_{1}, \mathrm{HH}_{2} \text { and } \mathrm{HH}_{3} \\
\boldsymbol{w}_{\boldsymbol{i}}=\boldsymbol{U}_{\boldsymbol{j}} \boldsymbol{S}_{\boldsymbol{j}} \boldsymbol{V}_{\boldsymbol{j}}^{\boldsymbol{T}} \mathrm{j}=\text { Patient image, Doctor image and Fingerprint }
\end{gathered}
$$

5. Apply step 1 and 4 to watermarked medical image to obtain:

$$
A_{\text {wati }}=\boldsymbol{U}_{\text {wati }} \boldsymbol{S}_{\text {wati }} \boldsymbol{V}_{\text {wati }}^{\boldsymbol{T}} \mathrm{i}=\mathrm{HH}_{1}, \mathrm{HH}_{2} \text { and } \mathrm{HH}_{3}
$$

6. The singular values of 'Patient image', 'Doctor image' and 'Fingerprint' are obtained by the singular values of $\mathrm{HH}_{3} \mathrm{HH}_{2}$, and $\mathrm{HH}_{1}$ of the watermarked medical image and the cover medical image respectively by (11):

$$
S_{j}^{\prime}=\left(S_{w a t i}-S_{i}\right) / \alpha
$$

7. The extracted watermark images 1 'Patient image' is obtained by applying ISVD using equation (11) and then applies inverse DCT and inverse DWT.

8. The extracted watermark image 2 'Doctor image' and the extracted watermark image 3 'Fingerprint' are obtained by applying ISVD using equation (11) and then apply inverse DWT.

\section{Experimental results}

The proposed approach DWT-DCT-SVD was simulated using Matlab R2013b.

a number of tests are applied on three cover medical images 'Brain', 'CT scan', and 'Knee' of size '512 $\mathrm{x}$ 512 ' and 'Patient image', 'Doctor image' and 'Fingerprint' of size 512x512 taken as watermark images shown in Figure 3.

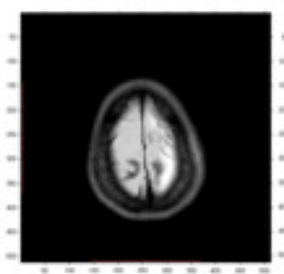

(a)

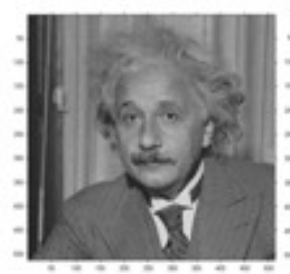

(e)

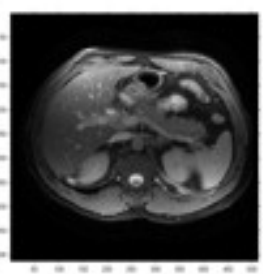

(b)

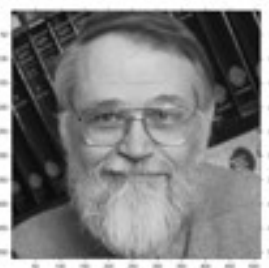

(f)

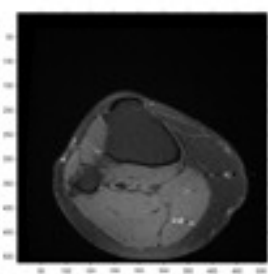

(c)

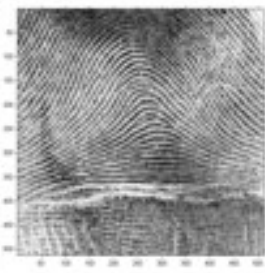

(g)

Figure 3. (a, b, c) Cover medical images: a) Brain. b) CT scan. c) Knee, (e, f, g) watermark images : e). Patient image. f) Doctor image. G) Fingerprint 
To evaluate the imperceptibility of our algorithm we have calculated the PSNR between the cover medical images and the watermarked images. Figure 4 approves that the proposed method gives an excellent quality of watermarked image.

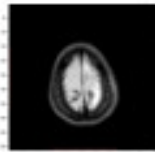

(a)

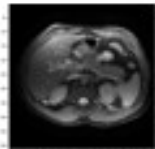

(f)

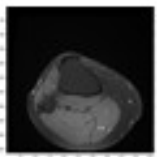

(k)

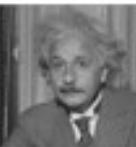

(b)

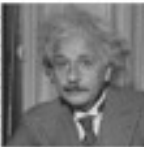

(g)

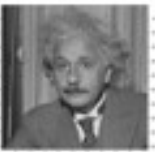

(1)

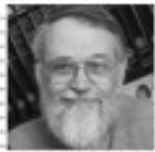

(c)

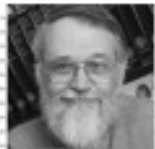

(h)

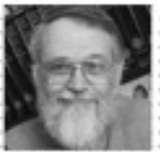

(m)

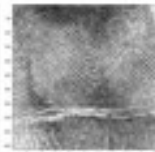

(d)

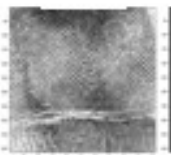

(i)

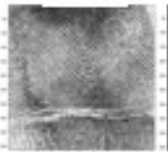

(n)

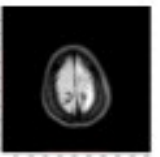

(e)

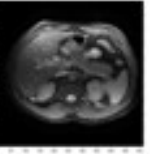

(j)

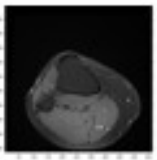

(o)

Figure 4. (a, $f, k)$ cover medical images. (b, $g$, l) watermark 1 'Patient image'. (c, h, m) watermark 2 'Doctor image'. (d, i, n) watermark 3 'Fingerprint'.

$(e, j, 0)$ watermarked images

The Tables 1, 2 and 3 show the quality of perceptibility of the proposed method without any noise attacks. When the cover medical image is 'Brain', The maximum of PSNR value obtained against the three watermark images 'Patient image', 'Doctor image ' and 'Fingerprint' is 42.70 at scaling factor 0.1 and minimum PSNR value is 36.84 at scaling factor 2 . In the case where the cover image is 'CT scan' the maximum PSNR value is 37.30 at scaling factor 0.1 and the minimum PSNR value is 33.02 at scaling factor 2 and with the cover medical image 'Knee' the maximum PSNR is 40.11 at scaling 0.1 and minimum PSNR value is 33.53 at scaling factor 2 . On the other hand, the NC values are 1 in all scaling factor shown in Tables 1, 2 and 3.

Table 1. PSNR and NC Performance of the Proposed Method for medical image Brain

\begin{tabular}{ccccc}
\hline \multirow{5}{*}{$\begin{array}{c}\text { Gain } \\
\text { factor }\end{array}$} & PSNR & $\begin{array}{c}\text { NC } \\
\text { (Patient } \\
\text { image) }\end{array}$ & $\begin{array}{c}\text { NC } \\
\text { (Doctor } \\
\text { image) }\end{array}$ & $\begin{array}{c}\text { NC } \\
\text { (Fingerprint) }\end{array}$ \\
\hline 0.1 & 42.70 & 1 & 1 & 1 \\
0.2 & 42.19 & 1 & 1 & 1 \\
2 & 36.84 & 1 & 1 & 1 \\
\hline
\end{tabular}


Transactions on Machine Learning and Artificial Intelligence Vol 5 No 4, Aug 2017

Table 2. PSNR and NC Performance of the Proposed Method for medical image CT scan

\begin{tabular}{ccccc}
\hline \multirow{4}{*}{$\begin{array}{c}\text { Gain } \\
\text { factor }\end{array}$} & \multicolumn{4}{c}{ CT scan } \\
\cline { 2 - 5 } & PSNR & $\begin{array}{c}\text { NC } \\
\text { (Patient } \\
\text { image) }\end{array}$ & $\begin{array}{c}\text { NC } \\
\text { (Doctor } \\
\text { image) }\end{array}$ & $\begin{array}{c}\text { NC } \\
\text { (Fingerprint) }\end{array}$ \\
\hline 0.1 & 37.30 & 1 & 1 & 1 \\
0.2 & 36.96 & 1 & 1 & 1 \\
2 & 33.02 & 1 & 1 & 1 \\
\hline
\end{tabular}

The PSNR values obtained by the proposed algorithm give better results than other techniques $[3,2$, and 6]. At scaling factor 0.1 the maximum PSNR value is 42.70 compared to 32.09 obtained by [3], 33.08 obtained by [2] and 32.19 obtained by [6]. When scaling factor is 0.2 , the maximum PSNR value is 42.19 compared to 27.29 obtained by [3] and finally at scaling factor 2, the maximum PSNR value is 36.84 compared to 27.78 obtained by [2].

Table 3. PSNR and NC Performance of the Proposed Method for medical image Knee

\begin{tabular}{ccccc}
\hline \multirow{4}{*}{$\begin{array}{c}\text { Gain } \\
\text { factor }\end{array}$} & KSNR & $\begin{array}{c}\text { NC } \\
\text { (Patient } \\
\text { image) }\end{array}$ & $\begin{array}{c}\text { NC } \\
\text { (Doctor } \\
\text { image) }\end{array}$ & $\begin{array}{c}\text { NC } \\
\text { (Fingerprint) }\end{array}$ \\
\hline 0.1 & 40.11 & 1 & 1 & 1 \\
0.2 & 39.44 & 1 & 1 & 1 \\
2 & 33.53 & 1 & 1 & 1 \\
\hline
\end{tabular}

To evaluate the robustness of our approach technique, various attacks were applying such as Gaussian noise, Speckle noise, Salt \& pepper and Median filtering. Table 4 shows the performance of NC values at scaling factor 0.2 .

The maximum NC value obtained against Gaussian noise, Speckle noise, Salt \& pepper and Median filtering is 1 for watermark image 'Doctor image'. However, the minimum NC value is 0.9571 against Speckle noise for watermark image 'Fingerprint'. 
Imane Assini, Abdelmajid Badri, Khadija Safi, Aicha Sahel, Abdennaceur Baghdad. Robust Multiple Watermarking Technique for Medical Applications using DWT, DCT and SVD. Transactions on Machine Learning and Artificial Intelligence, Vol 5 No 4 August (2017); p: 463-472

Table 4. Performance of NC values at gain factor $=0.2$

\begin{tabular}{|c|c|c|c|c|c|c|c|c|c|}
\hline \multirow[b]{2}{*}{ Attacks } & \multicolumn{3}{|c|}{ Brain } & \multicolumn{3}{|c|}{ CT Scan } & \multicolumn{3}{|c|}{ Knee } \\
\hline & $\begin{array}{c}\text { NC } \\
\text { Patient } \\
\end{array}$ & $\begin{array}{c}\text { NC } \\
\text { Doctor }\end{array}$ & $\begin{array}{c}\text { NC } \\
\text { Fingerprint }\end{array}$ & $\begin{array}{c}\text { NC } \\
\text { Patient }\end{array}$ & $\begin{array}{c}\text { NC } \\
\text { Doctor }\end{array}$ & $\begin{array}{c}\text { NC } \\
\text { Fingerprint }\end{array}$ & $\begin{array}{c}\text { NC } \\
\text { Patient } \\
\end{array}$ & $\begin{array}{c}\mathrm{NC} \\
\text { Doctor }\end{array}$ & $\begin{array}{c}\text { NC } \\
\text { Fingerprint }\end{array}$ \\
\hline $\begin{array}{c}\text { Gaussian } \\
\text { noise } \\
(\mathrm{M}=0, \mathrm{~V}=0.0 \\
0003)\end{array}$ & $\begin{array}{c}0.995 \\
6\end{array}$ & 1 & 0.9997 & 0.9988 & 1 & 0.9999 & $\begin{array}{c}0.999 \\
7\end{array}$ & 1 & 0.9998 \\
\hline $\begin{array}{c}\text { Gaussian } \\
\text { noise } \\
(\mathrm{M}=0, \mathrm{~V} \\
=0.001)\end{array}$ & $\begin{array}{c}0.995 \\
9\end{array}$ & 1 & 0.9840 & 0.9985 & 1 & 0.9748 & $\begin{array}{c}0.999 \\
4\end{array}$ & 1 & 0.9728 \\
\hline $\begin{array}{l}\text { Gaussian } \\
\text { noise } \\
\text { (M=0.01,V } \\
=0.0005)\end{array}$ & $\begin{array}{c}0.996 \\
2\end{array}$ & 1 & 0.9906 & 0.9991 & 1 & 0.9880 & $\begin{array}{c}0.999 \\
6\end{array}$ & 1 & 0.9863 \\
\hline $\begin{array}{c}\text { Salt \& pepper } \\
\text { (density = } \\
0.001)\end{array}$ & $\begin{array}{c}0.997 \\
2\end{array}$ & 1 & 0.9874 & 0.9991 & 1 & 0.9904 & $\begin{array}{c}0.999 \\
6\end{array}$ & 1 & 0.9870 \\
\hline $\begin{array}{c}\text { Salt \& pepper } \\
\text { (density = } \\
0.002 \text { ) }\end{array}$ & $\begin{array}{c}0.997 \\
1\end{array}$ & 1 & 0.9662 & 0.9991 & 1 & 0.9735 & $\begin{array}{c}0.999 \\
0\end{array}$ & 1 & 0.9687 \\
\hline $\begin{array}{c}\text { Speckle noise } \\
\text { (density = } \\
0.0002 \text { ) }\end{array}$ & $\begin{array}{c}0.996 \\
1\end{array}$ & 1 & 0.9996 & 0.9989 & 1 & 1 & $\begin{array}{c}0.999 \\
8\end{array}$ & 1 & 1 \\
\hline $\begin{array}{c}\text { Speckle noise } \\
\text { (density = } \\
0.005)\end{array}$ & $\begin{array}{c}0.997 \\
2\end{array}$ & 1 & 0.9957 & 0.9993 & 1 & 0.9934 & $\begin{array}{c}0.999 \\
9\end{array}$ & 1 & 0.9968 \\
\hline $\begin{array}{c}\text { Speckle noise } \\
\text { (density = } \\
0.02 \text { ) }\end{array}$ & $\begin{array}{c}0.998 \\
2\end{array}$ & 1 & 0.9691 & 0.9996 & 1 & 0.9571 & $\begin{array}{c}0.999 \\
8\end{array}$ & 1 & 0.9769 \\
\hline $\begin{array}{l}\text { Median } \\
\text { filtering }\end{array}$ & $\begin{array}{c}0.987 \\
7\end{array}$ & 1 & 0.9979 & 0.9826 & 1 & 0.9990 & $\begin{array}{c}0.992 \\
6\end{array}$ & 1 & 0.9985 \\
\hline
\end{tabular}

In Table 5, we have compared the robustness of our technique against various attacks compared to existing techniques [2], [3] and [10].

The maximum NC value obtained at scaling factor $=0.2$ is 1 for watermark image 'Doctor image' against Gaussian noise compared to 0.9908 obtained by [2], 0.9466 obtained by [3] and 0.944001 obtained by [10] respectively, 1 for watermark image 'Doctor image' against Salt \& pepper compared to 0.9953 obtained by [2], 0.9658 obtained by [3] and 0.949754 obtained by [10] respectively, 1 for watermark images 'Doctor image' and 'Fingerprint' against Speckle noise (density $=0.00001$ )compared to 0.949742obtained by [10], 1 for watermark image 'Doctor image' against Median filtering compared to 0.9861 obtained by [2], 0.0123 obtained by [3] and 0.9985 obtained by [10] respectively, 1 for watermark image 'Doctor image' against Salt \& Peppers $(d=0.002)+$ Speckle $(V=0.005)$ compared to 0.8328 obtained by [3], and finally 1 for watermark image 'Doctor image' against Gaussian ( $M=0.01, V=0.002)+$ Speckle $(\mathrm{V}=0.005)$ compared to 0.8276 obtained by [3]. 
Table 5. Comparison of the proposed method with existing approaches at scaling factor 0.2

\begin{tabular}{|c|c|c|c|c|c|c|c|}
\hline \multirow{2}{*}{ Attacks } & \multicolumn{3}{|c|}{ Proposed method } & \multicolumn{2}{|c|}{ [2] } & \multirow[t]{2}{*}{ [3] } & \multirow[t]{2}{*}{ [10] } \\
\hline & $\begin{array}{c}\text { NC } \\
\text { Patient }\end{array}$ & $\begin{array}{c}\text { NC } \\
\text { Doctor }\end{array}$ & $\begin{array}{c}\mathrm{NC} \\
\text { Fingerprint }\end{array}$ & $\begin{array}{c}\text { NC } \\
\text { Symptoms }\end{array}$ & $\begin{array}{c}\text { NC } \\
\text { Record }\end{array}$ & & \\
\hline $\begin{array}{c}\text { Gaussian noise } \\
(\mathrm{M}=0, \mathrm{~V}=0.00003)\end{array}$ & 0.9997 & 1 & 0.9998 & Not shown & $\begin{array}{c}\text { Not } \\
\text { shown }\end{array}$ & $\begin{array}{c}\text { Not } \\
\text { shown }\end{array}$ & 0.944001 \\
\hline $\begin{array}{l}\text { Gaussian noise } \\
(\mathrm{M}=0, \mathrm{~V}=0.001)\end{array}$ & 0.9994 & 1 & 0.9728 & 0.9515 & 0.9908 & 0.9466 & $\begin{array}{l}\text { Not } \\
\text { shown }\end{array}$ \\
\hline $\begin{array}{c}\text { Salt \& pepper } \\
\text { (density }=0.001 \text { ) }\end{array}$ & 0.9996 & 1 & 0.9870 & 0.9636 & 0.9953 & 0.9658 & 0.949754 \\
\hline $\begin{array}{c}\text { Speckle noise } \\
\text { (density }=0.00001)\end{array}$ & 0.9998 & 1 & 1 & Not shown & $\begin{array}{l}\text { Not } \\
\text { shown }\end{array}$ & $\begin{array}{l}\text { Not } \\
\text { shown }\end{array}$ & 0.949742 \\
\hline $\begin{array}{c}\text { Speckle noise } \\
\text { (density }=0.02 \text { ) }\end{array}$ & 0.9998 & 1 & 0.9769 & Not shown & $\begin{array}{c}\text { Not } \\
\text { shown }\end{array}$ & 0.8673 & $\begin{array}{l}\text { Not } \\
\text { shown }\end{array}$ \\
\hline Median Filtering & 0.9926 & 1 & 0.9990 & 0.8657 & 0.9861 & 0.0123 & 0.9985 \\
\hline $\begin{array}{c}\text { Salt \& Peppers } \\
(d=0.002)+ \\
\text { Speckle }(V=0.005)\end{array}$ & 0.9989 & 1 & 0.9615 & Not shown & $\begin{array}{c}\text { Not } \\
\text { shown }\end{array}$ & 0.8328 & $\begin{array}{l}\text { Not } \\
\text { shown }\end{array}$ \\
\hline $\begin{array}{c}\text { Gaussian } \\
(\mathrm{M}=0.01, \mathrm{~V}=0.002)+ \\
\text { Speckle }(\mathrm{V}=0.005)\end{array}$ & 0.9991 & 1 & 0.9284 & Not shown & $\begin{array}{c}\text { Not } \\
\text { shown }\end{array}$ & 0.8276 & $\begin{array}{l}\text { Not } \\
\text { shown }\end{array}$ \\
\hline
\end{tabular}

\section{Conclusion}

This article presents an innovative multiple watermarking method based on the combination of three techniques DWT, DCT, and SVD. Three watermark images 'Patient image', 'Doctor image' and 'Fingerprint' are embedded into a cover medical image of modalities: computer tomography (CT) and magnetic resonance imaging (MRI). The insertion is made in the high-frequency sub bands $\mathrm{HH} 1, \mathrm{HH} 2$ and $\mathrm{HH} 3$ of the cover medical image. However, the insertion in these areas makes it possible to enhance the robustness of the system of watermarking without damaging the visual quality of the watermarked image. The results obtained approve the performance of the proposed technique in terms of imperceptibility, capacity and robustness compared to existing techniques in the literature. In the future works, we will optimize and increase the quality of our algorithm by using the genetic algorithm.

\section{REFERENCES}

[1] Perednia DA, Allen A, "Telemedicine Technology and Clinical Applications".JAMA273(6):483-488,(1995) doi:10.1001/jama.1995.03520300057037. 
Imane Assini, Abdelmajid Badri, Khadija Safi, Aicha Sahel, Abdennaceur Baghdad. Robust Multiple Watermarking

Technique for Medical Applications using DWT, DCT and SVD. Transactions on Machine Learning and Artificial Intelligence, Vol 5 No 4 August (2017); p: 463-472

[2] Zear, Aditi, Amit Kumar Singh, and Pardeep Kumar. "Multiple Watermarking for Healthcare Applications." Journal of Intelligent Systems (2016).

[3] Zear, Aditi, Amit Kumar Singh, and Pardeep Kumar. "A proposed secure multiple watermarking technique based on DWT, DCT and SVD for application in medicine." Multimedia Tools and Applications (2016): 1-20.

[4] Singh, Siddharth, Vivek Singh Rathore, and Rajiv Singh. "Hybrid NSCT domain multiple watermarking for medical images." Multimedia Tools and Applications (2016): 1-19.

[5] Muharemagic, Edin, and Borko Furht. "Survey of watermarking techniques and applications." Multimedia Watermarking Techniques and Applications 3 (2006): 91-130.

[6] Singh, Amit Kumar, Mayank Dave, and Anand Mohan. "Hybrid technique for robust and imperceptible multiple watermarking using medical images." Multimedia Tools and Applications 75.14 (2016): 8381-8401.

[7] V. Santhi, N. Rekha, S. Tharini, "A Hybrid Block Based Watermarking Algorithm using DWT-DCT-SVD Techniques for Color Images" Computing, Communication and Networking. International Conference on IEEE Xplore 18-20 Dec. 2008

[8] I.Assini, A.Badri, K.Safi "Adaptation of Different Techniques on Digital Image Watermarking in Medical Domain: A review", International Journal of Advanced Research in Computer Science and Software Engineering (IJARCSSE), Volume 5, Issue 12, December 2015.

[9] Takore, Tamirat Tagesse, P. Rajesh Kumar, and G. Lavanya Devi. "A modified blind image watermarking scheme based on DWT, DCT and SVD domain using GA to optimize robustness." Electrical, Electronics, and Optimization Techniques (ICEEOT), International Conference on. IEEE, 2016.

[10] Sharma, Abhilasha, Amit Kumar Singh, and Satya Prakash Ghrera. "Robust and Secure Multiple Watermarking for Medical Images." Wireless Personal Communications (2016): 1-14 